\section{Expanding role of local anaesthesia in vitreoretinal surgery}

MTJ Costen', RS Newsom', AC Wainwright ${ }^{2}$, AJ Luff' and CR Canning'

Keywords: vitreoretinal; local; anaesthesia;

\author{
Purpose Local anaesthesia (LA) is \\ increasingly common in vitreoretinal surgery. \\ However, younger patients often have such \\ surgery under general anaesthesia (GA). \\ We reanalysed the anaesthetic practice for \\ vitreoretinal surgery in our unit over a \\ 19-month period.
}

Abstract

Methods A total of 1003 patients undergoing vitreoretinal surgery between August 2000 and February 2002 were studied. Type of surgery, patient pain score to anaesthesia and surgery, need for sedation and incidence of complications related to the local anaesthetic were recorded. Comparisons were made between this case series and previous data from our unit.

Results In total, 920/1003 $(91.7 \%)$ patients had LA. Total operations comprised 418

vitrectomies, 518 retinopexies with or without vitrectomy and 67 buckling procedures. More patients under the age of 35 years had LA than previously (60.2 vs $35.7 \%, P<0.001)$. In $920 / 920$ $(\mathbf{1 0 0 \% )}$ of cases, LA was administered via intraconal injection, compared to $164 / 1221$ (13.4\%) of procedures previously. Significantly more patients under the age of 35 years required sedation $(35.9 \%)$ than did older patients (19.2\%). Overall, use of sedation was significantly increased since our previous study (20.2 vs $\mathbf{7 . 8 \%}$ ). Anaesthesia and surgery were well tolerated by patients. There were no cases of orbital haemorrhage or ocular perforation. Complications included bradycardia requiring atropine $1 / 920(0.1 \%)$ and chemosis $88 / 920$

(9.6\%).

Conclusions LA is well tolerated and effective even in younger patients. Sedation may well be required in younger patients and for procedures involving scleral buckling. The main indication for GA was patient preference. Despite this, such patients accounted for only $5.2 \%$ of the total. Eye (2005) 19, 755-761. doi:10.1038/sj.eye.6701640; published online 24 September 2004

\author{
retrobulbar; intraconal; sedation; age
}

\section{Introduction}

Local anaesthesia (LA) for vitreoretinal surgery is generally well tolerated. ${ }^{1-3}$ Advantages include avoidance of general anaesthetic risks, good postoperative analgesia, ${ }^{4}$ and faster postoperative rehabilitation, in a predominantly daycase service. Peribulbar anaesthesia has been shown to be equally effective as retrobulbar anaesthesia in providing adequate analgesia and akinesia, ${ }^{5}$ reducing the risk of optic nerve sheath penetration from a long needle. Anaesthetic agent administered using a blunt cannula via the subtenons' route has also proved effective. ${ }^{6,7}$ The placement of a temporary indwelling retrobulbar catheter in the subtenons' space has been used to provide per- and postoperative titratable anaesthesia with high levels of patient satisfaction. . $^{8}$ However, for younger patients and for more involved vitreoretinal procedures, general anaesthesia (GA) has often been preferred over LA. ${ }^{10}$

We previously analysed our anaesthetic practice for vitreoretinal surgery within our department, where there has been a significant trend towards LA over the last 2 years. During a 6-year period up to July 2000 (2000 series), 82\% of patients had surgery under LA, but younger patients were more likely to have GA. ${ }^{1}$

We present the results of a case series from August 2000 (2001 series), highlighting changes in practice.

\section{Methods}

A total of 1003 consecutive patients undergoing vitreoretinal surgery between August 2000 and February 2002 were studied prospectively. All patients were anaesthetized by one anaesthetist $(\mathrm{ACW})$. Patient data were acquired at the time
${ }^{1}$ Southampton Eye Unit Southampton General Hospital

Southampton, UK

${ }^{2}$ Shackleton Department of Anaesthetics

Southampton General Hospital

Southampton, UK

\section{Correspondence:}

MTJ Costen

Southampton Eye Unit Southampton General Hospital

Tremona Road

Southampton SO16 6YD

UK

Tel.: +442380777222

Fax: + 442380794120

E-mail: mtcosten@

doctors.org.uk

Received: 9 November 2003 Accepted: 1 March 2004 Published online: 24 September 2004 
of operation and recorded on a laptop PC in the anaesthetic room.

LA comprised a 50/50 mix of $2 \%$ lignocaine and $0.75 \%$ bupivacaine with $1500 \mathrm{U}$ of hyalase and $1 \mathrm{ml}$ of sodium bicarbonate, to reduce the discomfort of injection. ${ }^{1}$

Approximately $0.2 \mathrm{ml}$ of $0.2 \%$ lignocaine was injected to form a small subconjunctival bleb near the injection site. The main injection was given inferotemporally, through conjunctiva, into the muscle cone (intraconal) in all cases.

Sedation in the form of intravenous midazolam was offered to patients on the judgement of the operating surgeon and the anaesthetist, after discussion with the patient. An amount of $2-4 \mathrm{mg}$ of midazolam was given as a single intravenous bolus, with the aim of inducing 'light' sedation. Sleep was prevented wherever possible, by conversing with the patient, so as to minimize the risk of unpredictable patient movements. Peroperative injection of $2 \%$ lignocaine was administered into the subtenons' space when there were signs of some patient discomfort. All patients in the current series received the anaesthetic via an intraconal route with or without supplementation of LA via medial injection and subtenons' top-up. Comparisons were made between the results of both of our studies. In relation to patient experience of LA and surgery, comparisons were made between all patients in this series and only those receiving intraconal LA previously. The patient's age, volume of anaesthetic, and need for sedation were noted.

For the purposes of the study, surgery was divided into three groups comprising vitrectomy, retinopexy (argon laser or cryotherapy \pm vitrectomy), and scleral buckling. Patients' response to the anaesthetic was assessed using a standard pain scale $1-4$ : (1, no pain; 2 , sensation, but no pain; 3 , mild response to pain; and 4 , strong response to pain). The pain of operation was graded as follows: 1 , no pain; 2 , minor (would not put off LA again); 3, moderate pain; and 4, severe pain (no local again). The surgeon was asked to grade akinesia immediately after the surgery. The grading was as follows: 1 , no movement; 2 , slight movement; $3,50 \%$ of full movement; and 4, full movement.

In order to ensure that surgical outcomes remained acceptable during the audit period, a retrospective audit of primary success of retinal detachment surgery was carried out. This revealed a primary reattachment rate of $88 \%$.

\section{Technique of intraconal injection}

The aim is to deliver the injection into the muscle cone via an anterior retrobulbar technique, as described by Rao et al. ${ }^{2}$ The patient is asked to fixate on a target to maintain the eyes in primary position. A 1-in 25-G needle is inserted with the bevel towards the globe, through bulbar conjunctiva, immediately below lateral rectus. The needle is passed posteriorly along the globe until the hub of the needle is in the same horizontal plane as the limbus. At this point, the needle is directed superomedially in the orbit. The plunger is withdrawn slightly to ensure that the needle is not within a blood vessel and the injection is slowly given. Confirmation of intraconal placement of the anaesthetic is purely observational, based on lowering of the upper lid. After approximately $5 \mathrm{ml}$ has been injected, the needle is slowly withdrawn along the same course. In some cases, a further injection of anaesthetic is given along the medial orbital wall if the operation is judged likely to be prolonged, involving significant manipulation of the globe or the application of a scleral buckle. Patients experiencing any discomfort during surgery were offered either topical or top-up subtenons' anaesthesia (2\% lignocaine) depending on the phase of surgery. Patients were regularly given additional subtenons' lignocaine prior to buckling surgery.

\section{Statistical methods}

Data were compared between different groups in this patient series and direct comparisons were made to our practice previously. Data were analysed using the 'Statview' software. Proportions were analysed using a $\chi^{2}$-test for nonparametric data. Parametric data were analysed using a paired $t$-test.

\section{Results}

In this series, 920/1003 (91.7\%) patients had vitreoretinal surgery performed under LA compared with $82.3 \%$ in a previous study $(P<0.001)$. All $920(100 \%)$ patients received their main local anaesthetic block via an intraconal route, compared to $164 / 1221$ (13.4\%) patients previously (Table $1 ; P<0.001$ ).

Vitreoretinal procedures under LA comprised 405/920 (44\%) vitrectomies, 479/920 (52.1\%) retinopexies with or without vitrectomy, and 36/920 (3.9\%) procedures involving scleral buckling. Of the patients undergoing retinopexy, $327 / 479(68.3 \%)$ had laser (indirect or endo-) and $152 / 479(31.7 \%)$ had cryotherapy.

Patients under 35 years of age were more likely to have a local anaesthetic than general anaesthetic 53/88 (60.2\%; 95\% CI: 55-65.4). This is a significant change from our previous study where only $46 / 129(35.7 \%)$ of those under 35 years had LA (95\% CI: 31.4-39.9; $P<0.001$ ) (Figure 1 ). Younger patients were more likely to complain of some discomfort on injection, 17/53 (32.1\%: 95\% CI: 25.7-38.5) than older patients 105/867 (12.1\%: 95\% CI: 11-13.2; $P<0.01)$. We found a similar difference in our previous study with 15/47 (31.9\%: 95\% CI: 25.1-38.7) younger 
Table 1 Showing patient demographics and type of anaesthesia

\begin{tabular}{|c|c|c|c|c|c|}
\hline & \multicolumn{2}{|c|}{2001} & \multicolumn{2}{|c|}{2000} & \multirow[t]{2}{*}{$\mathrm{P}$} \\
\hline & $\%$ & $95 \% C I(\%)$ & $\%$ & $95 \% C I(\%)$ & \\
\hline Patients & 100 & & & 100 & \\
\hline \multicolumn{6}{|l|}{ Type of anaesthetic } \\
\hline LA & 91.7 & & 82.3 & & $<0.001$ \\
\hline Intraconal & 100 & & 13.4 & & \\
\hline Peribulbar & & & 10.6 & & \\
\hline Combined intraconal and peribulbar & & & 75.9 & & \\
\hline GA & 8.3 & & 17.7 & & $<0.001$ \\
\hline Under 35 years & 8.8 & & 8.7 & & \\
\hline LA & 60.2 & $55-65.4$ & 35.7 & $31.4-39.9$ & $<0.001$ \\
\hline GA & 39.8 & $34.6-45$ & 64.3 & & \\
\hline Sedation (total no.) & 20.2 & $18.9-21.5$ & 7.8 & $7-8.6$ & $<0.001$ \\
\hline$<35$ years & 35.9 & $29.3-42.4$ & 10.6 & $6.1-10.1$ & $<0.05$ \\
\hline$>35$ years & 19.2 & 17.9-20.6 & 7.7 & $6.9-8.4$ & $<0.001$ \\
\hline \multicolumn{6}{|l|}{ Type of operation } \\
\hline Vitrectomy & 41.7 & $40.1-43.2$ & 35.7 & $33.4-38$ & \\
\hline Retinopexy $+/-$ vitrectomy & 51.7 & $50.1-53.2$ & 44.7 & $42.5-46.7$ & \\
\hline Scleral buckling & 6.7 & $5.9-7.5$ & 19.5 & $16.9-22.1$ & $<0.001$ \\
\hline
\end{tabular}

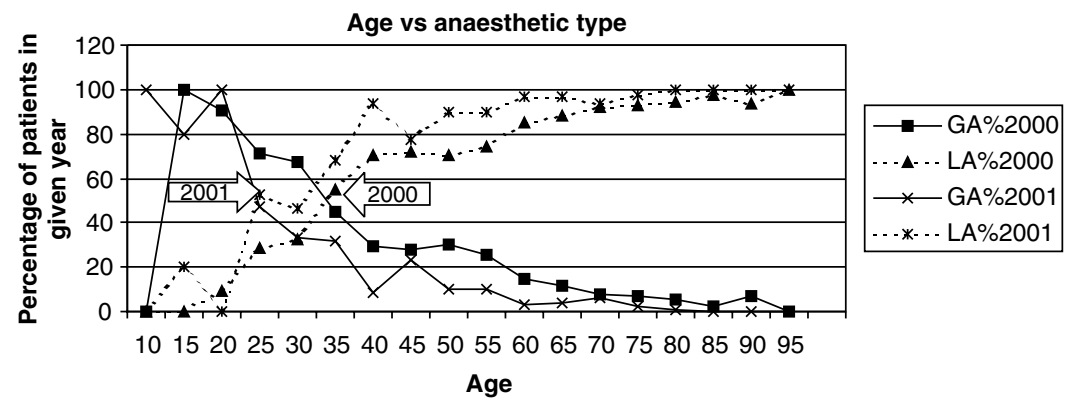

Figure 1 Showing age $v$ s type of anaesthetic. Note that the change from predominantly GA to LA occurs approximately 10 years earlier than in the 2000 series (arrows).

patients complaining of discomfort compared with 176/ 1174 (15\%: 95\% CI: 14-16) patients over 35 years of age.

\section{Intraconal anaesthesia}

Patients in our previous study who received intraconal injection alone comprised $164(13.4 \%)$ patients. Such patients were significantly more likely to complain of discomfort on injection $(33.6 \%, 95 \%$ CI: 29.3-36.6) than those in our current series $(13.3 \%, 95 \%$ CI: $12.1-14.4$, $P<0.01$ (Figure 2 and Table 2).

Considering the total volume of local anaesthetic injected, average total volumes for vitrectomy were $8.46 \mathrm{ml}$ (SD $1.11 \mathrm{ml}$ ) and for retinopexy with or without vitrectomy: $8.72 \mathrm{ml}$ (SD $1.14 \mathrm{ml}) P=0.1$ (Table 2). For buckling procedures, the volume was significantly greater than for the other procedures $10.65 \mathrm{ml}$

(SD $1.92 \mathrm{ml}) P<0.01$. In our previous study, total volumes for vitrectomy and retinopexy in the intraconal group were $9.11 \mathrm{ml}(1.15 \mathrm{ml})$ and $13.17 \mathrm{ml}(3.51 \mathrm{ml})$ respectively, which were significantly greater than in our current series $(P<0.01)$.

Top-up anaesthesia by subtenons' injection was required peroperatively in 10/405 (2.5\%; 95\% CI: $1.7-3.2)$ patients undergoing vitrectomy; $19 / 479$ (4\%; 95\% CI: 3.1-4.9) patients undergoing retinopexy and 15/36 (41.7\%; 95\% CI: 33.5-49.9, $P<0.001)$ patients undergoing scleral buckling (Table 3). In our previous study, a similar number of patients required top-up for vitrectomy 8/141 (5.7\% ; 95\% CI: 3.7-7.6) $P>0.1$, but more patients required top-up for retinopexy 16/23 (69.6\%; 95\% CI: 60-79.2) $P<0.01$. 


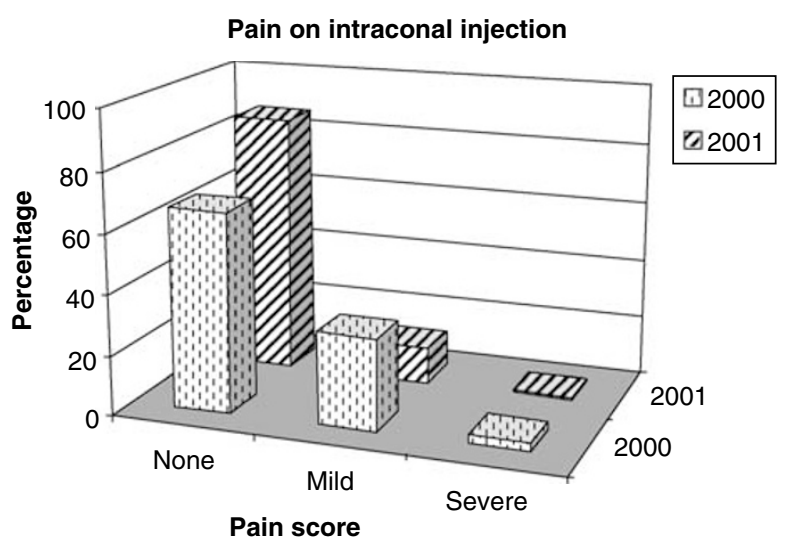

Figure 2 Percentage of patients experiencing discomfort on intraconal injection. Pain scores for both 'no pain' and 'sensation only' have been grouped together for this figure.

\section{Peroperative pain scores}

Pain scores of 1,2,3,4 were reported by $838 / 920$ (91.1\%; 95\% CI: 90.2-92), 73/920 (7.9\%; 95\% CI: 7-8.8), 9/920 (1\%; 95\% CI: $0.7-1.3)$, and $0 \%$ patients, respectively. In our previous study of intraconal LA, the pain scores were 150/164 (91.5\%; 95\% CI: 89.3-93.7, P>0.5) 13/164 (7.9\%; 95\% CI: $5.8-10, P>0.5), 0 / 164$ and $1 / 164(0.6 \%$; $95 \%$ CI: $0-1.2)$, respectively. There was no statistical difference in overall peroperative pain scores between the two studies (Table 2).

Patients undergoing scleral buckling surgery were no more likely to complain of significant pain during surgery $(0 / 36)$ than patients undergoing vitrectomy (6/ 405) $(1.5 \%$; 95\% CI: $0.9-2.1)$ or retinopexy $3 / 479(0.6 \%$; 95\% CI: 0.3-1.0). Comparing cryotherapy with laser, no

Table 2 Anaesthetic and operation pain scores and volume of anaesthetic agent injected

\begin{tabular}{|c|c|c|c|c|c|}
\hline & \multicolumn{2}{|c|}{2001} & \multicolumn{2}{|c|}{2000} & \multirow[t]{2}{*}{$\mathrm{P}$} \\
\hline & $\%$ & $95 \% C I(\%)$ & $\%$ & $95 \% C I(\%)$ & \\
\hline \multicolumn{6}{|c|}{ Pain on intraconal injection } \\
\hline No pain & 74.5 & $73-75.9$ & 3.1 & $1.7-4.4$ & $<0.01$ \\
\hline Sensation & 12.1 & $11-13.1$ & 63.4 & $59.7-67.2$ & $<0.01$ \\
\hline Mild pain & 12.3 & $11.2-13.4$ & 30.5 & $26.9-34.1$ & $<0.01$ \\
\hline Severe pain & 1 & $0.7-1.3$ & 3.1 & $1.7-4.4$ & $<0.05$ \\
\hline \multicolumn{6}{|l|}{ Pain of operation } \\
\hline No pain & 91.1 & $90.2-92$ & 91.5 & $89.3-93.7$ & $>0.5$ \\
\hline Sensation & 7.9 & $7-8.8$ & 7.9 & $5.8-10$ & $>0.5$ \\
\hline Mild pain & 1 & $0.7-1.3$ & 0 & & \\
\hline Severe pain & 0 & & 0.6 & $0-1.2$ & \\
\hline \multicolumn{6}{|c|}{ Volume of anaesthetic ( $\mathrm{ml}$ ) } \\
\hline Vitrectomy & $8.46(1.11)$ & & $9.11(1.15)$ & & 0.1 \\
\hline Retinopexy & $8.72(1.14)$ & & $13.17(3.51)$ & & \\
\hline Scleral buckle & $10.65(1.92)$ & & & & $<0.001$ \\
\hline
\end{tabular}

Table 3 Showing the incidence of supplemental anaesthesia, degree of kinesia and chemosis

\begin{tabular}{|c|c|c|c|c|c|}
\hline & \multicolumn{2}{|c|}{2001} & \multicolumn{2}{|c|}{2000} & \multirow[t]{2}{*}{$\mathrm{P}$} \\
\hline & $\%$ & $95 \% C I(\%)$ & $\%$ & $95 \% C I(\%)$ & \\
\hline \multicolumn{6}{|c|}{ Need for top-up anaesthesia $(\%, 95 \%$ CI) } \\
\hline Vitrectomy & 2.5 & $1.7-3.2$ & 5.7 & $3.7-7.6$ & $>0.1$ \\
\hline Retinopexy $+/-$ vitrectomy & 4 & $3.1-4.9$ & 69.6 & $60-79.2$ & $<0.01$ \\
\hline Buckle & 41.7 & $33.5-49.9$ & & & \\
\hline Any kinesia & 3.2 & $1.7-3.2$ & 39 & $35-42.8$ & $<0.01$ \\
\hline Chemosis & 9.6 & $8.6-10.5$ & & & \\
\hline \multicolumn{6}{|l|}{ Mean age of patients (years) } \\
\hline All & 61.2 & Range 10-91 & & & \\
\hline LA & 62 & Range 14-91 & 63.6 & Range $20-103$ & \\
\hline GA & 41.8 & Range $10-75$ & 45.9 & Range 10-87 & \\
\hline
\end{tabular}




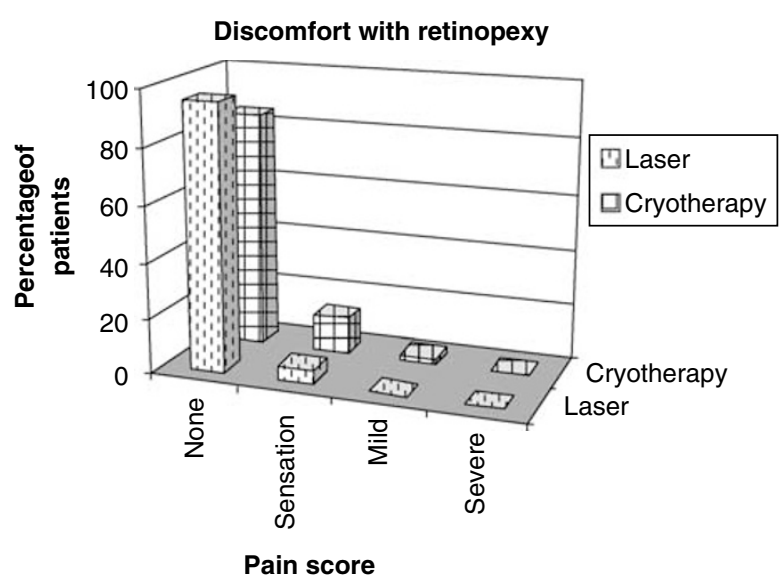

Figure 3 Pain scores for both laser and cryotherapy.

patients reported significant pain (Figure 3). Significantly more patients undergoing cryotherapy reported some awareness of surgery: 20/152 (13.2\%; 95\% CI: 10.4-15.9) than did those undergoing laser: $16 / 329$ (4.9\%; 95\% CI: 3.7-6.1, $P<0.001)$. Reporting of significant pain in our previous study was similar for both vitrectomy $1 / 141$ (0.7\%; 95\% CI: $0-1.4)$ and for retinopexy $0 / 23$.

\section{Sedation}

Sedation was given to $186 / 920$ patients $(20.2 \%$; $95 \%$ CI: $18.9-21.5 \%)$. This is significantly different from our previous study where 95/1221 (7.8\%; 95\% CI: 7-8.6\%; $P<0.001)$ patients were given sedation. Younger patients (under 35 years) were more likely to be given sedation before LA; $19 / 53$ (35.9\%; 95\% CI: 29.3-42.4) than older patients $167 / 868$ (19.2\%; 95\% CI: 17.9-20.6; $P<0.05)$. In comparison with our previous study, there has been a significant increase in the proportion of younger patients being given sedation: $35.9 \%$ this series compared to $10.6 \%$ previously $(P<0.05)$ (Table 1$)$. Sedation was used in $67 / 405(16.5 \%$; $95 \%$ CI: $14.7-18.4 \%)$ vitrectomies, in 100/479 (20.9\%; 95\% CI: 19-22.7\%) patients undergoing retinopexy with or without vitrectomy and 19/36 (52.8\%; 95\% CI: 44.5-61.1\%) patients undergoing scleral buckling (Figure 4).

\section{Kinesia}

There were no reports of significant kinesia. Overall, there were significantly fewer reports of any kinesia in the current series compared with previous (Figure 5 and Table 3). Slight movement was noted in 12/405 patients (3\%; 95\% CI: $2.1-3.8 \%)$ undergoing vitrectomy and 17/479 patients $(3.6 \%$; 95\% CI: $2.7-4.4)$ undergoing retinopexy. In our previous study of intraconal LA, slight movement was noted in 53/141 patients $(37.6 \%$; $95 \%$

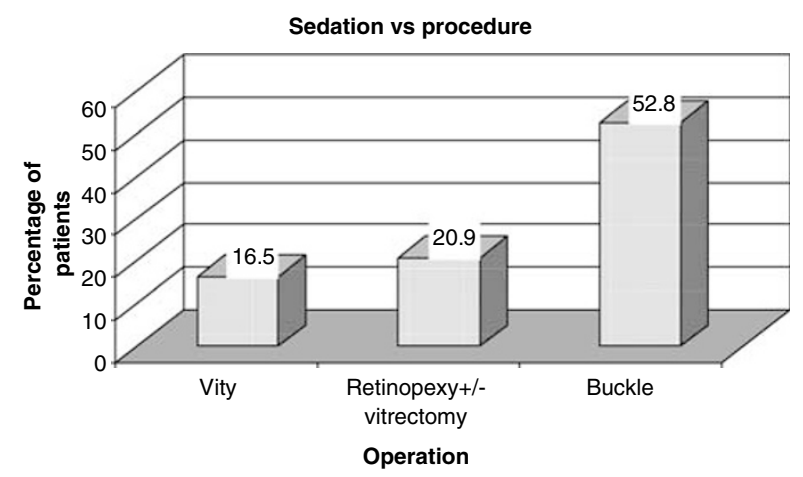

Figure 4 The percentage of patients receiving sedation for each of the procedures in the 2001 series.

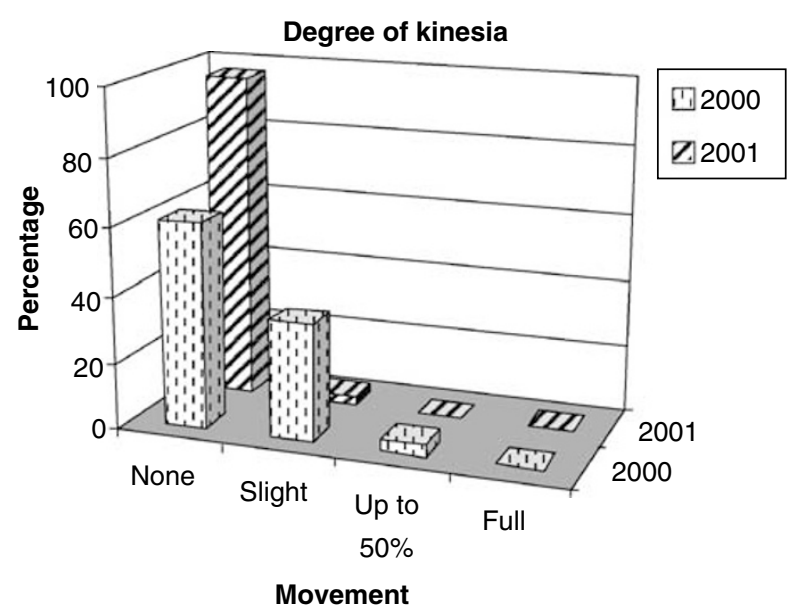

Figure 5 Degree of movement (kinesia) for all procedures having intraconal anaesthesia for 2000 and 2001.

CI: 33.5-41.7; $P<0.01)$ undergoing vitrectomy and 10/24 patients $(41.7 \%$; 95\% CI: 31.6-51.7\%; $P<0.01)$ undergoing retinopexy.

\section{Complications}

There were no cases of globe perforation during this period. In total, $88 / 920$ patients $(9.6 \%$; $95 \%$ CI: $8.6-10.5 \%)$ had moderate to severe chemosis, none was severe enough to prevent surgery taking place. In total, 1/920 patients $(0.1 \%$; 95\% CI: $0-0.24 \%)$ had bradycardia severe enough to warrant atropine injection.

\section{Indications for $G A$}

The most common indication of GA in this series was patient preference $(62.7 \%$ ) (Figure 6). The other main indications were the need for bilateral surgery in one session $(18.1 \%)$ and patients' age (7.2\%). There were only four $(4.8 \%)$ patients who underwent GA on the basis of the nature of the surgery. In total, $31 / 67$ (46.2\%, 95\% 


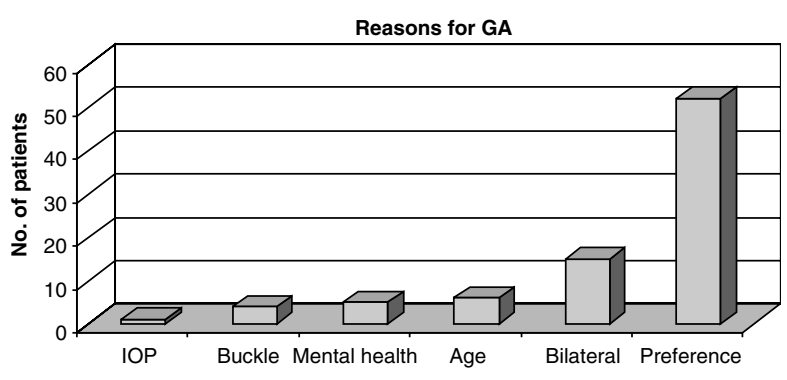

Figure 6 Reasons for GA (IOP denotes GA chosen due to concerns about raised intraocular pressure secondary to LA). Numbers indicate percentages.

CI: 40.2-52.3) patients undergoing scleral buckling had GA compared with 39/518 (6\%, 95\% CI: 4.9-7.0; $P<0.001)$ patients undergoing retinopexy with or without vitrectomy. No patients had GA on the basis of axial length of the eye or because of concomitant warfarin administration.

\section{Discussion}

We present the findings of a large series of patients (1003) undergoing vitreoretinal procedures. The proportion of patients having LA for such procedures has increased in our unit from $82.3 \%$ in the 6 years up to 2000 , to $91.7 \%$ more recently. In addition, more younger patients have had such surgery under LA than previously: $60.2 \mathrm{vs}$ $35.7 \%$. Our use of sedation has increased, particularly for younger patients. Overall, $20.2 \%$ of patients were sedated in this series compared to $7.8 \%$ previously. These percentages are both higher than that reported in the National Audit of LA for cataract surgery in 1999. ${ }^{11}$ The decision to use sedation is made jointly by the anaesthetist, the patient, and the surgeon prior to the anaesthetic. Factors such as the length, the likely complexity of the operation, and the perceived anxiety levels of the patient being important factors. Such a trend in usage is largely based on experience. There were no significant adverse events related to the sedation noted in our study.

There has been a trend in our unit towards universal administration of anaesthetic agent into the muscle cone. This is supplemented by a medial injection and possibly a subtenons' top-up for procedures likely to be more prolonged and uncomfortable, such as the use of cryotherapy or the application of a scleral buckle. There are concerns regarding the use of a sharp needle so close to the globe. ${ }^{12}$ However, there were no serious complications related to the injection in our study. Chemosis was noted in 88 (9.6\%) patients, but in none of these did the chemosis hinder surgery.

A clear 'learning curve' can be seen in our experience of intraconal LA, with fewer patients experiencing discomfort on injection this series compared to previous, reduced anaesthetic volumes and fewer reports of any kinesia. Good akinesia is obviously more important for certain vitreoretinal procedures over others. This has often led to the choice of a general anaesthetic rather than local for some procedures such as membrane peeling. Our experience with this technique of LA is of excellent akinesia in the vast majority of cases. One concern regarding the widespread use of LA in vitreoretinal surgery is its possible effect on teaching. We have not found the use of LA to be a hindrance to surgical teaching in our unit.

The use of GA does permit examination and, if necessary, treatment of fellow eye lesions at the same session. However, such patients can readily be examined as an outpatient and appropriate treatment given.

From our study of LA for vitreoretinal surgery within our unit, patient tolerability remains high. With the increase in the use of LA for vitreoretinal procedures, it is important to ensure that this remains acceptable and safe for patients. In-patient beds are increasingly under pressure and hence general anaesthetic services are often stretched. Theatre flexibility and the ability to add urgent 'nonstarved' patients to the list are other clear advantages of a predominantly local anaesthetic service. In our study, there were very few contraindications to LA. No patients were selected for GA on the basis of axial length or warfarin administration. Choice of GA over LA was predominantly due to patient preference.

Therefore, the overall low GA rate in our series is largely due to careful preoperative counselling by an anaesthetist experienced in ophthalmic LA.

Interestingly, a significant proportion of the patients having GA had procedures involving scleral buckling $(46.3 \%)$.

In our unit, we have noted a trend towards fewer scleral buckling procedures, with more patients undergoing primary vitrectomy for retinal detachment and the use of tamponade agents with posturing for more complex vitreoretinal situations, which would previously have required local scleral buckling or encirclement. Clearly, with an increasing number of procedures performed under LA, it is important to ensure that clinical outcomes remain acceptable. An audit of retinal detachment surgery during the 19-month study period revealed a primary reattachment rate of $88 \%$, exceeding the minimum standard proposed in the National Audit of Retinal Detachment Surgery. ${ }^{13}$

From our study, it would appear that LA is well tolerated and effective even in younger patients. Careful patient selection, together with the use of sedation when necessary, should ensure that routine use of LA for vitreoretinal surgery continues to become more acceptable to patients and medical staff alike. 


\section{References}

1 Newsom RS, Wainwright AC, Canning CR. Local anaesthesia for 1221 vitreoretinal procedures. $\mathrm{Br} J$ Ophthalmol 2001; 85: 225-227.

2 Rao GP, Wong D, Groenewald C, McGalliard JN, Jones A, Ridges PJ. Local anaesthesia for vitreoretinal surgery: a case-control study of 200 cases. Eye 1998; 12 (Part 3a): 407-411.

3 Sharma T, Gopal L, Parikh S, Shanmugam MP, Badrinath SS, Mukesh BN. Parabulbar anesthesia for primary vitreoretinal surgery. Ophthalmology 1997; 104: 425-428.

4 Kristin N, Schonfeld CL, Bechmann M, Bengisu M, Ludwig $\mathrm{K}$, Scheider A et al. Vitreoretinal surgery: pre-emptive analgesia. Br J Ophthalmol 2001; 85: 1328-1331.

5 Demediuk OM, Dhaliwal RS, Papworth DP, Devenyi RG, Wong DT. A comparison of peribulbar and retrobulbar anesthesia for vitreoretinal surgical procedures. Arch Ophthalmol 1995; 113: 908-913.

6 Kwok AK, Van Newkirk MR, Lam DS, Fan DS. Sub-Tenon's anesthesia in vitreoretinal surgery: a needleless technique. Retina 1999; 19: 291-296.
7 Stevens JD, Franks WA, Orr G, Leaver PK, Cooling RJ. Fourquadrant local anaesthesia technique for vitreoretinal surgery. Eye 1992; 6 (Part 6): 583-586.

8 Jonas JB, Budde WM, Dinkel M, Hemmerling TM. Indwelling temporary retrobulbar catheter for long-lasting titratable local anesthesia. Arch Ophthalmol 2000; 118: 996-1000.

9 Jonas JB, Hemmerling TM, Budde WM, Dinkel M. Postoperative analgesia by reinjections of local anesthetic through an indwelling retrobulbar catheter. Am J Ophthalmol 2000; 129: 54-58.

10 Newsom R, Luff A, Wainwright C, Canning C. UK survey of attitudes to local anaesthesia for vitreoretinal surgery. Eye 2001; 15: 708-711.

11 Eke T, Thompson JR. The National Survey of Local Anaesthesia for Ocular Surgery. I. Survey methodology and current practice. Eye 1999; 13 (Part 2): 189-195.

12 Waller SG, Taboada J, O'Connor P. Retrobulbar anesthesia risk. Do sharp needles really perforate the eye more easily than blunt needles? Ophthalmology 1993; 100: 506-510.

13 National audit of the outcome of primary surgery for rhegmatogenous retinal detachment. II. Clinical outcomes. Eye 2002; 1(6): 771-777. 